\title{
Treatment outcomes of MDR-TB and HIV co-infection in Europe
}

\author{
To the Editor:
}

The ongoing HIV epidemic and the increasing number of patients with drug-resistant tuberculosis (TB) are seriously hampering global TB-control activities, including those in the World Health Organization (WHO) Region Europe. Overall, the prevalence of HIV co-infection in TB patients increased from $3.4 \%$ in 2008 to $8 \%$ in 2014 in the region [1]. The prevalence of multidrug-resistant (MDR)-TB (drug resistance against at least isoniazid and rifampicin) reported for Europe - $15 \%$ in newly diagnosed TB patients and $48 \%$ in previously treated TB patients - is the highest in the world [1].

Data on MDR-TB and HIV co-infection in Europe are scarce. Recently, surveillance data on HIV infection in patients with MDR-TB were published [2]. A meta-analysis on treatment outcomes for 2587 adult HIV-infected patients with MDR-TB reported a pooled on-treatment mortality risk of $38 \%$ and treatment success for $49.9 \%$ [3]. The contribution of data from countries in the WHO Europe region was very limited.

The WHO definition for treatment success in MDR-TB [4] is driven by treatment completion rather than being relapse-free in the absence of therapy after treatment completion. Recently, TBnet proposed simplified MDR-TB outcome definitions that include a post-treatment relapse-free observation period of 1 year for a definition of cure [5].

The aim of the current study is to assess the effect of HIV infection on treatment outcomes in patients with MDR-TB in WHO region Europe, applying these new outcome definitions.

We used the data collected in the TBnet MDR-TB cohort [5-7]. Between January 2010 and November 2011, patients with culture-confirmed MDR-TB were consecutively enrolled at 23 MDR-TB treatment centres in 16 European countries [6]. Follow-up time was from the time of diagnosis until a WHO-defined outcome or 12 months after cure or treatment completion. MDR-TB treatment outcome was evaluated by current WHO guidelines [4] and the following new definitions [5].

1) Cure. Negative culture status 6 months after treatment initiation, no positive culture thereafter, and no relapses within 1 year of treatment completion.

2) Treatment failure. Positive culture status 6 months after treatment initiation or thereafter, or a relapse within 1 year of treatment completion.

3) Undeclared outcome. Outcome that was not assessed (owing to transfer out of the cohort, no culture status at 6 months while the patient was receiving care, or no post-treatment assessment).

4) Death. Death during observation.

5) Loss to follow-up. Non-receipt of care 6 months after treatment initiation.

Early treatment responses and late treatment outcomes for all enrolled patients were compared by HIV status (positive versus negative/unknown). Early treatment response, based on culture conversion rate within 6 months of start of treatment, was calculated with the Kaplan-Meier estimate. The effect of HIV co-infection was assessed by a Cox proportional hazard analysis after verifying that the proportional hazard assumption was met. Treatment success and relapse-free cure were compared by HIV status using logistic regression models that incorporated a robust standard error to allow for clustering of events within country of treatment. The level for statistical significance was set at 5\% in all analyses.

@ERSpublications

HIV-positive patients with MDR-TB demonstrate a very low proportion of relapse-free cure http://ow. ly/r9sk30bqLBP

Cite this article as: Magis-Escurra C, Günther G, Lange C, et al. Treatment outcomes of MDR-TB and HIV co-infection in Europe. Eur Respir J 2017; 49: 1602363 [https://doi.org/10.1183/13993003.023632016]. 


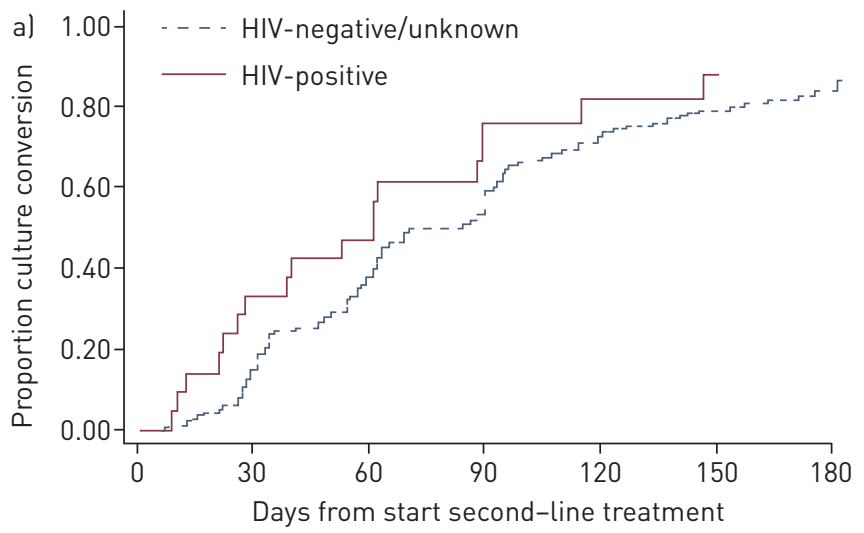

Simplified definitions

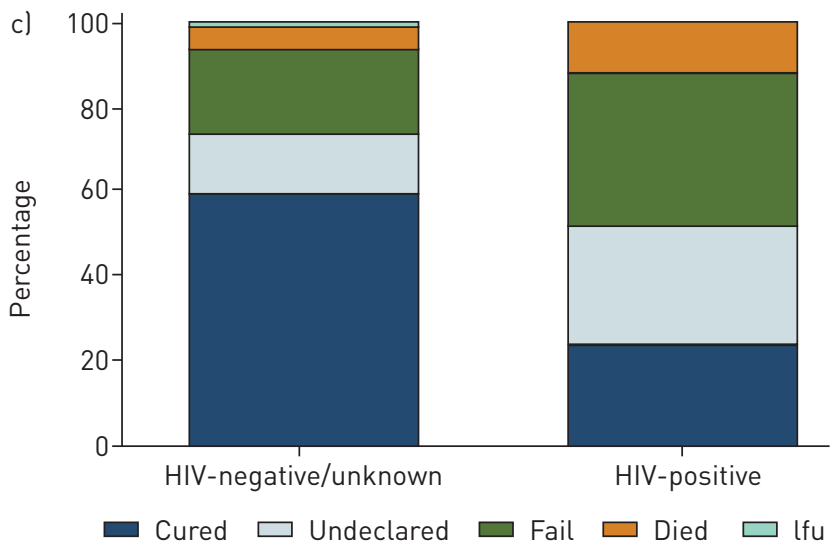

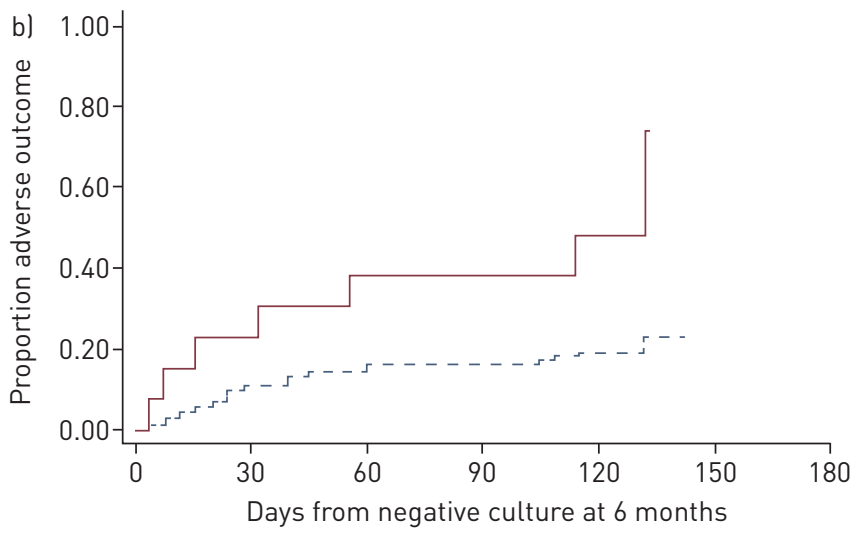

WHO definitions

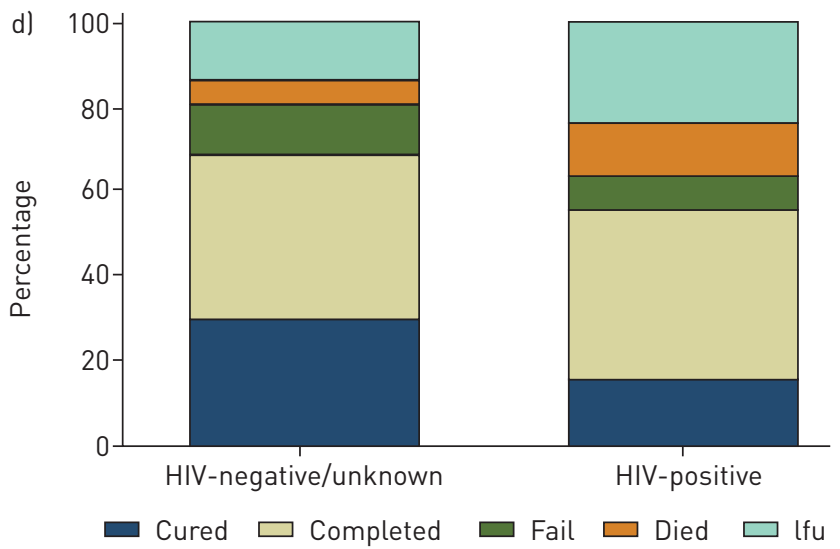

FIGURE 1 a) Culture conversion; time point 0 is the start of second-line treatment, including all patients. b) Any adverse outcome (failure or relapse); time point 0 is month 6 after start of second-line treatment, including all patients with a negative culture at 180 days after start of second-line treatment. Multidrug-resistant tuberculosis treatment outcomes by c) simplified and d) World Health Organization definitions. (fu: lost to follow-up

The study was approved by the ethical board of the University of Lübeck (Germany; ref. no. 09-106) and the responsible ethical review boards of all participating sites.

A total of 380 patients were included: 25 (6.6\%) were HIV-infected, 345 not infected and 10 patients were not tested. Thirteen (52\%) of the HIV-positive patients received antiretroviral therapy (ART). CD4 cell count at MDR-TB diagnosis was available for 16 (64\%) HIV-positive patients (median $183 \mathrm{cells} \cdot \mathrm{mm}^{-3}$, interquartile range 44-294).

Culture conversion was not negatively influenced by HIV status (fig. 1). The Kaplan-Meier estimates at 6 months after start of treatment for HIV-positive and HIV-negative/unknown patients were $88.1 \%$ and $84.0 \%$, respectively, with an associated hazard ratio (HR) of 1.48 (95\% CI 0.95-2.31).

Relapse-free cure was achieved in $24.0 \%$ of the HIV-positive patients, compared to $59.4 \%$ of the HIV-negative/unknown patients (odds ratio (OR) 0.22 , 95\% CI $0.10-0.46, \mathrm{p}<0.001$ ). With the WHO definition, an association between HIV status and late treatment outcome was also seen, although it was less strong. WHO-defined cure was reported in $16 \%$ of HIV-positive patients and $30.1 \%$ of HIV-negative/ unknown patients (OR 0.44, 95\% CI 0.18-1.11; $\mathrm{p}=0.083$ ).

The discrepancy between a high culture conversion rate and low frequency of relapse-free cure is driven by higher frequencies of adverse or undeclared outcomes (fig. 1). HIV-positive patients were significantly more likely to either fail or die in the period after the initial 6 months of treatment than HIV-negative/ unknown patients (HR 3.33; 95\% CI 1.50-7.36, p=0.03) (fig. 1).

Adverse events occurred with similar frequencies in HIV-positive and HIV-negative/unknown patients (data not shown). In both groups, $28 \%$ of the events initiated a temporary or permanent change of drugs (data not shown). 
The main finding of this study is an absent association between HIV status and early treatment response (culture conversion), but a negative association between HIV positivity and relapse-free cure. Using WHO-defined cure, the association with HIV status is not statistically significant. The simplified treatment outcome definitions seem to capture better the known association between TB-HIV co-infections and adverse outcome.

These findings on culture conversion and the negative impact of HIV infection on late treatment outcomes are in concordance with European surveillance data and observational studies $[3,8,9]$.

The differential trajectory of HIV-positive patients within the first 6 months of MDR-TB treatment and at treatment completion is striking. The higher prevalence of factors negatively influencing treatment, such as unemployment, injection drug use, smoking and co-infection with hepatitis B and/or C, either directly or through non-adherence to therapy, seem important.

The effect of ART on the prevention of mortality in TB-HIV co-infected patients is well established [10]. The study showed $12.0 \%$ mortality in HIV-positive patients, even though only $50 \%$ used ART in this cohort, in contrast with the $38 \%$ mortality in a meta-analysis [3]. The meta-analysis included many studies lacking information on ART-use and from other epidemiological settings than Europe. The use of ART may be under-reported in our study. The EuroCoord study group recently showed major differences between WHO regions Eastern and Western Europe in the organisation and delivery of healthcare for TB patients with HIV co-infection $[11,12]$. Our study endorses this claim. More attention needs to be paid to HIV testing in all TB patients, the timely initiation of ART, and intensifying follow-up.

A limitation of our study was the small number of MDR-TB patients with HIV co-infection among all patients with MDR-TB in the cohort, precluding stratification on ART use. The routine collection of cohort data may have contributed to the underreporting of adverse events. Due to this limitation, reported associations should be interpreted with care.

In conclusion, simplified MDR-TB outcome definitions capture the known association between MDR-TBHIV co-infection and poor treatment outcome better than the conventional WHO definitions. They demonstrate the very low proportion of patients with relapse-free cure of MDR-TB in HIV co-infected patients. Findings from this study are important for the clinical care of patients with MDR-TB and HIV co-infection, as they indicate that adequate treatment response at 6 months of MDR-TB therapy should not lead to complacency by the treating physician, given the higher risk of adverse outcomes throughout the remaining treatment period.

Cecile Magis-Escurra ${ }^{1,35}$, Gunar Günther ${ }^{2,3,35}$, Christoph Lange ${ }^{2,3,4,5,35}$, Sofia Alexandru ${ }^{6}$, Neus Altet ${ }^{7}$, Korkut Avsar ${ }^{8}$, Didi Bang, Raisa Barbuta ${ }^{10}$, Graham Bothamley ${ }^{11}$, Ana Ciobanu ${ }^{6}$, Valeriu Crudu ${ }^{6,12}$, Manfred Davilovits ${ }^{13}$, Martin Dedicoat ${ }^{14,15}$, Raquel Duarte ${ }^{16,17}$, Gina Gualano ${ }^{18}$, Heike Kunst ${ }^{19,20}$, Wiel de Lange $^{21,22}$, Vaira Leimane ${ }^{23}$, Anne-Marie McLaughlin ${ }^{24}$, Inge Muylle ${ }^{25}$, Veronika Polcováa ${ }^{26}$, Christina Popa ${ }^{27}$, Rudolf Rumetshofer ${ }^{28}$, Alena Skrahina ${ }^{29}$, Varvara Solodovnikova ${ }^{29}$, Victor Spinu ${ }^{27}$, Simon Tiberi ${ }^{20,30,31}$, Piret Viiklepp ${ }^{32}$ and Frank van Leth ${ }^{33,34,35}$ for TBnet

${ }^{1}$ Radboud University Medical Centre - TB Expert Centre UCCZ Dekkerswald, Nijmegen-Groesbeek, The Netherlands.

${ }^{2}$ Research Center Borstel, Clinical Infectious Diseases, German Center for Infection Research (DZIF), Borstel, Germany.

${ }^{3}$ Dept of Medicine, University of Namibia School of Medicine, Windhoek, Namibia. ${ }^{4}$ International Health/Infectious Diseases, University of Lübeck, Lübeck, Germany. ${ }^{5}$ Dept of Medicine, Karolinska Institute, Stockholm, Sweden. ${ }^{6}$ Institute of Phthisiopneumology, Chisinau, Moldova. ${ }^{7}$ Hospital Universitari Vall d'Hebron, Research Institute-IDIAP Jordi Gol, Barcelona, Spain. ${ }^{8}$ Asklepios Klinik Gauting, Gauting, Germany. ${ }^{9}$ Statens Serum Institut, Copenhagen, Denmark. ${ }^{10}$ Balti Municipal Hospital, Balti, Republic of Moldova. ${ }^{11}$ Homerton University Hospital, London, UK.

${ }^{12}$ National TB Reference Laboratory, Chisinau, Republic of Moldova. ${ }^{13}$ Dept of Respiratory Medicine, Tartu University Lung Hospital, Tartu, Estonia. ${ }^{14}$ Heart of England Foundation Trust, Birmingham, UK. ${ }^{15}$ University of Warwick, Coventry, UK. ${ }^{16}$ EpiUnit, Institute of Public Health, Porto University, Porto, Portugal. ${ }^{17}$ Dept of Clinical Epidemiology, Predictive Medicine and Public Health, University of Porto Medical School, Porto, Portugal. ${ }^{18}$ National Institute for Infectious Diseases "L. Spallanzani" (INMI), Rome, Italy. ${ }^{19}$ Dept of Medicine, Queen Mary University, London, UK.

${ }^{20}$ Barts Health NHS Trust, London, UK. ${ }^{21}$ University of Groningen, University Medical Center Groningen, Groningen, The Netherlands. ${ }^{22}$ Tuberculosis Centre Beatrixoord, Haren, The Netherlands. ${ }^{23}$ Riga East University Hospital, Tuberculosis and Lung Diseases Centre, Riga, Latvia. ${ }^{24}$ St James's Hospital, Dublin, Ireland. ${ }^{25}$ Dept of Respiratory Medicine, University Medical Center St Pieter, Brussels, Belgium. ${ }^{26}$ Thomayer University Hospital, Prague, Czech Republic. ${ }^{27}$ Marius-Nasta-Institut, Bucharest, Romania. ${ }^{28}$ Otto Wagner Hospital, Vienna, Austria. ${ }^{29}$ Clinical Dept, Republican Research and Practical Centre for Pulmonology and Tuberculosis, Minsk, Belarus. ${ }^{30} \mathrm{AOVV}$ E, Morelli Reference Hospital for MDR and HIV-TB, Sondalo, Italy. ${ }^{31}$ Dept of Infectious Diseases, Queen Mary University, London, UK. ${ }^{32}$ National Institute for Health Development, Tallinn, Estonia. ${ }^{33}$ Dept of Global Health, Academic Medical Center, University of Amsterdam, Amsterdam, The Netherlands. ${ }^{34}$ Amsterdam Institute for Global Health and Development, Amsterdam, The Netherlands. ${ }^{35}$ These authors contributed equally to this article.

Correspondence: Christoph Lange, Clinical Infectious Diseases, Research Center Borstel, Parkallee 35, 23845 Borstel, Germany. E-mail: clange@fz-borstel.de 
Received: Dec 22016 | Accepted after revision: Feb 222017

Support statement: This work was supported by the European Commission's Seventh Framework Programme (FP7/ 2007-2013) under grant agreement FP7-223681 (TB-PAN-NET). C. Lange is supported by the German Center for Infection Research (DZIF). Funding information for this article has been deposited with the Crossref Funder Registry.

Conflict of interest: Disclosures can be found alongside this article at erj.ersjournals.com

Acknowledgements: The authors thank Bram Prins (Amsterdam Institute of Global Health and Development, Amsterdam, The Netherlands) and Nelleke Smitsman (Research Center Borstel, Sülfeld, Germany) for valuable data managing support.

\section{References}

1 European Centre for Disease Prevention and Control/WHO Regional Office for Europe. Tuberculosis. Surveillance and Monitoring in Europe 2016. Stockholm, European Centre for Disease Prevention and Control, 2016.

2 Karo B, Krause G, Hollo V, et al. Impact of HIV infection on treatment outcome of tuberculosis in Europe. AIDS 2016; 30: 1089-1098.

3 Isaakidis P, Casas EC, Das M, et al. Treatment outcomes for HIV and MDR-TB co-infected adults and children: systematic review and meta-analysis. Int J Tuberc Lung Dis 2015; 19: 969-978.

4 World Health Organization. Definitions and Reporting Framework for Tuberculosis - 2013 Revision. Geneva, World Health Organization, 2013.

5 Günther G, Lange C, Alexandru S, et al. Treatment outcomes in multidrug-resistant tuberculosis. N Engl J Med 2016; 375: 1103-1105.

6 Günther G, van Leth F, Alexandru S, et al. Multidrug-resistant tuberculosis in Europe, 2010-2011. Emerg Infect Dis 2015; 21: 409-416.

7 Günther G, van Leth F, Altet N, et al. Beyond multidrug-resistant tuberculosis in Europe: a TBNET study. Int $J$ Tuberc Lung Dis 2015; 19: 1524-1527.

8 Post FA, Grint D, Werlinrud AM, et al. Multi-drug-resistant tuberculosis in HIV positive patients in Eastern Europe. J Infect 2014; 68: 259-263.

9 Podlekareva DN, Efsen AM, Schultze A, et al. Tuberculosis-related mortality in people living with HIV in Europe and Latin America: an international cohort study. Lancet HIV 2016; 3: e120-e131.

10 Odone A, Amadasi S, White RG, et al. The impact of antiretroviral therapy on mortality in HIV positive people during tuberculosis treatment: a systematic review and meta-analysis. PLoS ONE 2014; 9: e112017.

11 Efsen AM, Schultze A, Post FA, et al. Major challenges in clinical management of TB/HIV coinfected patients in Eastern Europe compared with Western Europe and Latin America. PLoS ONE 2015; 10: e0145380.

12 Mansfeld M, Skrahina A, Shepherd L, et al. Major differences in organization and availability of health care and medicines for HIV/TB coinfected patients across Europe. HIV Med 2015; 16: 544-552. 